\title{
Predictive value of serum collagen biomakers on the outcome of acute myocardial infarction treated with percutaneous coronary intervention
}

\author{
Hang Ga0 ${ }^{a}$, Xue-Song Zhang ${ }^{\mathrm{a}}$, Qiang Zhao ${ }^{\mathrm{b}}$, Long-Le Ma ${ }^{\mathrm{a}, \mathrm{c}}$, Le-Xin Wang ${ }^{\mathrm{a}, \mathrm{c}}$
}

\begin{abstract}
Aims. This study was designed to investigate the predictive value of serum collagen biomarkers on the outcomes of acute ST-elevation myocardial infarction (STEMI) treated with percutaneous coronary intervention (PCI).

Methods. Two hundred and ten patients with STEMI were successfully treated with PCI within 6 hrs ofthe onset of chest pain. The levels of serum procollagen type I carboxyterminal peptide (PICP) and procollagen type III peptide (PIIINP) were measured by enzymelinked immunosorbent assay (ELISA) before, 3 and 6 months after PCI. Left ventricular ejection fraction was assessed by echocardiography at 3 and 6 months after $\mathrm{PCl}$. The composite endpoints were death by any cause, recurrent myocardial infarction, heart failure or stroke.

Results. At the end of the 12-month follow up, 29 patients (13.8\%) experienced an end point. The level of serum PICP in patients with an end point was higher than in patients without an end point 7 days (19.45 $\pm 2.17 \mathrm{vs} 14.95 \pm 3.07 \mathrm{ng} /$ $\mathrm{mL}, P<0.05)$ or 3 month after the $\mathrm{PCl}(29.87 \pm 3.02 \mathrm{vs} 22.14 \pm 3.33 \mathrm{ng} / \mathrm{mL}, P<0.05)$. The serum PIIINP level in patients with an end point was also higher than those without 7 days after $\mathrm{PCl}(59.34 \pm 4.23 \mathrm{vs} 48.78 \pm 4.23 \mathrm{ng} / \mathrm{mL}, P<0.05)$. Multivariate logistic regression analysis showed day $7(\mathrm{OR}=2.170,95 \% \mathrm{Cl} 1.583-4.345, P=0.01)$ and 3-month serum $\mathrm{PICP}$ ( $O R=2.340,95 \% \mathrm{Cl}$ 1.431-4.650, $P=0.01$ ) were independent predictors of composite end points.

Conclusions. Persistent elevation of serum collagen marker PICP three months after PCI predicts an adverse outcome for patients with acute ST-elevation myocardial infarction.
\end{abstract}

Key words: myocardial infarction, percutaneous coronary intervention, procollagen type I carboxyterminal peptide, left ventricular function

Received: September 11, 2013; Accepted: November 27, 2013; Available online: December 11, 2013 http://dx.doi.org/10.5507/bp.2013.091

aDepartment of Cardiology, Liaocheng People's Hospital, Liaocheng 252000, PR China

${ }^{b}$ Department of Cardiology, the Red Cross Hospital of Guangzhou City, the Fourth Affiliated Hospital of Jinan University, Guangzhou 510220, PR China

'School of Biomedical Sciences, Charles Sturt University, Wagga Wagga, NSW 2678, Australia

Corresponding author: Lexin Wang, e-mail:/wang@csu.edu.au

\section{INTRODUCTION}

Acute ST-segment elevation myocardial infarction (STEMI) is a leading cause of morbidity and mortality. Early revascularization with percutaneous coronary intervention (PCI) or thrombolytic therapy is associated with significant clinical benefits, such as reduction in the size of infarction, improvement in left ventricular function and decreases in mortality ${ }^{1-3}$.

Ventricular remodeling is a major determining factor for the post-infarct ventricular function and the long-term prognosis following acute MI ( ref. $\left.^{4}\right)$. Type I collagen is a major component of myocardial extracellular matrix and plays a key role in ventricular remodeling ${ }^{4}$. Carboxyterminal peptide of type I procollagen (PICP), a serum biomarker of myocardial type I collagen synthesis, was elevated within the first few days following acute MI (ref. ${ }^{5,6}$ ). The other type of collagen that is associated with ventricular remodeling is collagen type III, which is a fibrillar constituent of granulation tissues. The aminoterminal propeptide of type III procollagen (PIIINP), a serum biomarker of myocardial biosynthesis of type III collagen, is associated with poor left ventricular function and high mortality following MI ( ref. $^{7}$ ).
Several earlier clinical observations in a small number of patients have found that coronary revascularization with PCI or thrombolytic therapy within the first $12 \mathrm{~h}$ of symptom onset diminished MI-induced PICP or PIIINP elevation $^{8,9}$. Although the reduction in PICP or PIIINP may represent less ventricular remodeling or dysfunction, the predictive value of post-PCI PICP and PIIINP on left ventricular function remains controversial ${ }^{8,9}$. In the present study, we investigated the relations between post-PCI levels of serum PICP and PIIINP and the clinical outcome in patients with STEMI.

\section{PATIENTS AND METHODS}

\section{Patient selection}

This study was approved by the institutional review board of Liaocheng People's Hospital. Informed written consent was obtained from all participants. Between January 2009 and November 2011, 210 consecutive patients (157 males, mean age 62.1 \pm 6.3 years) with new onset STEMI and presented within $6 \mathrm{~h}$ of the onset of chest pain were recruited into this study. In the same period, 364 patients with one or more of the following 
criteria were excluded: a) previous Q-wave MI; b) previous diagnosis of congestive heart failure or cardiomyopathy; c) severe valvular heart disease or severe left ventricular dysfunction (New York Heart Association Function class IV) on admission; d) chronic renal dysfunction; e) refusal to participate in this study; f) unsuccessful PCI.

The diagnosis of ST-elevation MI was based on typical angina chest pain, ST segment elevation on body surface ECG, and positive a troponin test.

\section{Study Protocol}

On admission, all patients received aspirin, clopidogrel and lipid-lowering drugs as standard management. All proceeded with PCI within 6 h of the onset of chest pain. PCI was performed using standard techniques. The selection of catheters, stents and adjunctive therapies was left to the discretion of the operators. Coronary angiography was performed through the right femoral approach. Stent (drug-eluting stent or bare metal stent) was implanted and direct stenting was allowed if suitable culprit lesion was visualized $^{6,9}$. Success of PCI was defined as TIMI flow grade 2 or 3 and residual stenosis $<50 \%$ at the culprit coronary artery.

\section{Measurement of PICP and PIIINP}

PICP and PIIINP were measured from venous blood samples collected before PCI, 7 days and 3 months after PCI. The post-PCI blood samples were collected between 8-9 am each morning. All samples were centrifuged immediately at $3000 \mathrm{rpm}$ for $10 \mathrm{~min}$ and plasma samples were stored at $-70{ }^{\circ} \mathrm{C}$ until assay. PICP and PIIINP were measured by enzymelinked immunosorbent assay (ELISA) (Fanbang Biotech, Dalian, China). The interassay coefficient of variation (CVs) for PICP and PIIINP were $1.2 \%$ and $1.6 \%$ at mean values of $1.7 \mathrm{ng} / \mathrm{mL}$ and $1.6 \mathrm{pg} / \mathrm{mL}$, respectively, and the intraassay coefficients of variation were $1.4 \%$ and $2.3 \%$ at mean values of $1.4 \mathrm{ng} / \mathrm{mL}$ and 1.3 $\mathrm{pg} / \mathrm{mL}$, respectively.

\section{Echocardiographic studies}

The left ventricular function was assessed with twodimensional and Doppler echocardiographic studies performed 3, 6 and 12 months after PCI (ViViD7 cardiac ultrasound unit, 2.5-MHz transducer, GE, PA, USA). The examiner of the echocardiography was not aware of the patient's clinical data. Left ventricular ejection fraction was measured from the apical views by Simpson's biplane method. The mean of 3 measurements was used.

\section{Follow-up}

Patients were followed-up monthly for 12 months after MI in our cardiology clinics. Data on medication compliance after discharge and occurrence of end points were recorded. Primary endpoint was death due to any cause. The secondary endpoints were recurrent $\mathrm{MI}, \mathrm{PCI}$ or coronary artery bypass grafting $(\mathrm{CABG})$, readmission for heart failure and stroke.

\section{Statistical analysis}

SPSS 13.0 was used for statistical analysis. Quantitative variables are expressed as mean $\pm \mathrm{SD}$. Categorical data were compared by Fisher's exact test. Comparisons of numerical data between groups were conducted by student t tests. ANOVA was used to compare the data collected from day 7, 3, and 6 months. Pearson correlation analysis was performed to assess the relations between the serum levels of PICP and PIIINP and the left ventricular ejection fraction. Multivariate logistic regression analysis was performed to identify predictors of adverse cardiac events. A $P$ value $<0.05$ was considered statistically significant.

\section{RESULTS}

\section{General finding}

PCI was successful in all patients. There were no mortalities during hospitalization and patients were discharged after an average of $3.2 \pm 2.1$ days of post-procedural hospital stay.

All patients completed the follow-up. One or more end points were observed in 29 patients (13.8\%). Four patients $(1.9 \%)$ died of sudden cardiac death between 6 to 12 months after the discharge. Eight patients (3.8\%) required target lesion revascularization. Recurrent nonfatal MI occurred in 6 (2.9\%), and newly developed heart failure required hospitalization was found in $9(4.3 \%)$. Stroke was found in $2(1.0 \%)$ patients.

As shown in Table 1, there was no statistically significant difference between patients with or without end points in age, sex, baseline cardiovascular risk factors, locations of MI and the number of coronary arteries involved at the initial presentation. The mean systolic blood pressure in patients with an end point was higher than in the patients without an end point $(P=0.02)$. There was no statistically significant difference in the post-PCI pharmacological therapies between the two groups $(P>0.05)$.

\section{Left ventricular function in patients with and without an end point}

There was no statistically significant difference in the left ventricular ejection fraction between patients with and without an end point at 3-month follow up (53.4 \pm $6.8 \%$ vs $58.7 \pm 8.5 \%, P>0.05)$. However, the mean left ventricular ejection fraction in patients with an end point was lower than in the patients without an end point at 6 months $(51.4 \pm 6.6$ vs $59.6 \pm 7.2 \%, P<0.05)$ and 12-month $(51.5 \pm 9.1 \%$ vs $57.2 \pm 9.4 \%, P<0.05)$ follow-up. Pearson correlation analysis showed that the level of PICP at day $7(\mathrm{r}=-0.630, P=0.001)$ and at 3 months $(\mathrm{r}=-0.671$, $P=0.001$ ) were negatively associated with left ventricular ejection fraction measured at 6 months following PCI. There was no statistically significant correlation between PIIINP and the post-PCI left ventricular ejection fraction $(P>0.05)$. 
Table 1. Comparison of baseline characteristics.

\begin{tabular}{lccc}
\hline & $\begin{array}{c}\text { With end points } \\
(\mathrm{n}=29)\end{array}$ & $\begin{array}{c}\text { Without end points } \\
(\mathrm{n}=181)\end{array}$ & $P$ \\
\hline Age (yrs) & $64.6 \pm 10.0$ & $62.7 \pm 9.4$ & NS \\
Sex (male, \%) & $18(62.1 \%)$ & $114(63.0 \%)$ & NS \\
Hypertension (\%) & $12(41.3 \%)$ & $79(43.6 \%)$ & NS \\
SBP (mmHg) & $139.2 \pm 12.0$ & $121.8 \pm 10.4$ & NS \\
DBP (mmHg) & $80.6 \pm 9.8$ & $79.5 \pm 10.2$ & NS \\
Diabetes (\%) & $9(31.0 \%)$ & $55(30.4 \%)$ & NS \\
Anterior MI (\%) & $20(68.9 \%)$ & $124(68.5 \%)$ & NS \\
Inferior MI (\%) & $9(31.1 \%)$ & $57(31.5 \%)$ & NS \\
Coronary lesions & $12(41.3 \%)$ & $76(42.0 \%)$ & \\
Single vessel & $9(31.0 \%)$ & $54(29.8 \%)$ & \\
Double vessel & $8(27.6 \%)$ & $51(28.2 \%)$ & \\
Three vessel & $18(100 \%)$ & $32(100 \%)$ & NS \\
Post-PCI therapies & $29(100 \%)$ & $181(100 \%)$ & NS \\
Aspirin (\%) & $28(96.6 \%)$ & $176(97.2 \%)$ & NS \\
Clopidogrel (\%) & $26(89.7 \%)$ & $164(90.6 \%)$ & NS \\
ACEI/ARB (\%) & $29(100 \%)$ & $181(100 \%)$ & NS \\
Beta-blockers (\%) & & & \\
Statins & & & \\
\hline
\end{tabular}

MI, myocardial infarction; SBP and DBP, systolic and diastolic blood pressure; PCI:

percutaneous coronary intervention; ACEI, angiotensin-converting enzyme inhibitor; ARB,

angiotensin II receptor blocker; NS, no significance.

Table 2. Comparison of PICP and PIIINP between the two groups.

\begin{tabular}{lcl}
\hline & PICP $(\mathrm{ng} / \mathrm{mL})$ & PIIIP $(\mathrm{pg} / \mathrm{mL})$ \\
\hline With an end point $(\mathrm{n}=29)$ & $11.01 \pm 2.19$ & $32.21 \pm 4.02^{*}$ \\
Pre-PCI & $19.45 \pm 2.17^{* * \boldsymbol{\Delta}}$ & $59.34 \pm 4.23^{* * \boldsymbol{\Delta}}$ \\
Day 7 & $29.87 \pm 3.02^{* \boldsymbol{\Delta}}$ & $49.67 \pm 6.93^{*}$ \\
3-month & $15.04 \pm 3.29^{*}$ & $36.87 \pm 7.45^{*}$ \\
6-month & & \\
Without an end point $(\mathrm{n}=181)$ & $10.76 \pm 2.60$ & $30.44 \pm 4.96$ \\
Pre-PCI & $14.95 \pm 3.07^{* *}$ & $48.78 \pm 4.23^{* *}$ \\
Day 7 & $22.14 \pm 3.33^{*}$ & $40.93 \pm 6.94$ \\
3-month & $13.56 \pm 2.53$ & $31.57 \pm 5.68^{*}$ \\
6-month
\end{tabular}

${ }^{* *} P<0.01$ compared with the before percutaneous coronary intervention $(\mathrm{PCI})$ value in the same group; ${ }^{*} P<0.05$, compared with Day 7 in the same group; ${ }^{\wedge} P<0.05$, compared with the patients without an end point.

\section{Comparison of PICP and PIIINP in patients with and without an end point}

As shown in Table 2, in patients with and without an end point, the day 7 serum PICP and PIIINP were higher than the pre-PCI levels within each group $(P<0.001)$. The mean level of serum PICP at 3-month was higher than that at day 7 in the two groups $(P<0.05)$. At the end of 6 months, the mean level of PICP in patients with an end point was lower than at the day $7(P<0.05)$. In patients without an end point, there was no statistically significant difference in the mean PICP level between day 7 and 6 months $(P>0.05)$.

In both groups, the mean PIIINP level 6 months after the PCI was lower than that at day $7(P<0.05)$. The mean day 7 level of PIIINP in patients with an end point was higher than in the patients without $(P<0.05)$.

\section{Predictive value of PICP and PIIINP on composite end points}

Multivariate logistic regression analysis revealed that the predictors of adverse cardiovascular events were age ( $\mathrm{OR}=2.612$, 95\% CI, 1.315-4.850, $P=0.01$ ), left ventricular ejection fraction 3 months after PCI $(\mathrm{OR}=3.820,95 \%$ CI 1.9522-6.479, $P<0.001)$, day $7(\mathrm{OR}=2.170,95 \% \mathrm{CI}$ 1.583-4.345, $P=0.01$ ) and 3-month level of serum PICP $(\mathrm{OR}=2.340,95 \%$ CI 1.431-4.650, $P=0.01)$. PIIINP was not an independent predictor of the composite end point $(P>0.05)$. 


\section{DISCUSSION}

The main findings of this study are: a) There was an elevation in the serum PICP and PIIINP within the first three months of PCI in patients with STEMI; b) The day 7 and 3-month levels of PICP and PIIINP in patients who suffered from a major adverse cardiovascular event were higher than in patients without a major cardiovascular event 12 months after the PCI; c) Together with age and post-PCI left ventricular ejection fraction, the serum levels of PICP 7 days and 3 months after the PCI are an independent predictor of adverse cardiovascular events. These results suggest that post-PCI levels of PICP may be used to assess patient's risk and to facilitate post-procedural monitoring and management.

Postinfarction left ventricular remodeling is a complex process. Infarct expansion, which results in ventricular dilatation and thinning of the wall in the first few hours of MI, involves the degradation of the intermyocyte collagen struts triggered by serine proteases and matrix metalloproteinases ${ }^{10}$. Serum levels of PICP, an indicator for myocyte collagen degradation or turnover, were found to closely relate to left ventricular function and the prognosis of MI (ref. ${ }^{11}$ ). Matrix metalloproteinase-1, an enzyme responsible for extracellular collagen degradation and remodeling, was also elevated in the first two weeks following MI (ref. ${ }^{12}$ ). Matrix metalloproteinase- 1 measured 7 days and at 2 weeks of the MI was negatively correlated with the left ventricular ejection fraction ${ }^{12}$. In the present study, the serum PICP was elevated in patients with or without an end point, and the mean serum PICP levels at 3 months were higher than on day 7, suggesting the collagen turnover may have reached its peak approximately 3 months after the onset of MI. In patients with an end point, the mean left ventricular ejection fraction at 6 months was lower than in patients without a major adverse cardiovascular event. There was a negative correlation between the day 7 and 3-month serum PICP levels and the left ventricular ejection fraction 6 months after the PCI. Serum PICP levels measured 7 days and 3 months after PCI predicted the major adverse cardiovascular events 12 months after the PCI. These results indicate that a high level of serum PICP following successful PCI is associated with ventricular remodeling and deterioration of left ventricular function, and adverse cardiovascular outcomes.

The value of serum PIIINP in predicting the prognosis of MI is uncertain. Primary coronary revascularization within $6 \mathrm{~h}$ of MI was associated with a greater reduction of PICP than the revascularization performed more than 12 days after the MI ( ref. $^{9}$ ). However, the early coronary revascularization was not associated with a greater reduction in serum PIIINP (ref. ${ }^{9}$ ). A recent study on 233 patients with acute MI also failed to show any longitudinal changes or predictive values of serum PIIINP ( ref. $^{13}$ ). In the present study, although serum PIIINP levels were elevated following MI and PCI, they were not correlated with the left ventricular ejection fraction 3 or 6 months after PCI. Post-PCI serum levels of PIIINP did not predict the adverse cardiovascular events 12 months after the
PCI. These results suggest that although serum PIIINP may reflect post-MI ventricular remodeling, it is not a reliable indicator for post-MI ventricular function or adverse clinical outcomes.

In conclusion, serum cardiac collagen biomarkers were elevated following acute ST-elevation MI. Successful PCI was associated with a reduction in serum PICP and PIIINP. Persistent elevation of serum PICP within the 3 months following PCI was associated with left ventricular dysfunction, and major adverse cardiovascular events 12 months after the PCI.

\section{ACKNOWLEDGEMENT}

Author contributions: All autors contributed equally to preparing the manuscript.

Conflict of interest statement: None declared.

\section{REFERENCES}

1. Boersma E. Primary Coronary Angioplasty vs. Thrombolysis Group. Does time mater? A Pooled analysis of randomized clinical trials comparing primary percutaneous coronary intervention and in-hospital fibrinolysis in acute myocardial infarction patients. Eur Heart J 2006;27:779-88.

2. Flaherty JD, Davidson CJ, Faxon DP. Percutaneous coronary intervention for myocardial infarction with left ventricular dysfunction. Am J Cardiol 2008;102(5A):38G-41G.

3. Busk M, Kaltoft A, Nielsen SS, Bøttcher M, Rehling $M$, Thuesen L, Bøtker HE, Lassen JF, Christiansen EH, Krusell LR, Andersen HR, Nielsen TT, Kristensen SD. Infarct size and myocardial salvage after primary angioplasty in patients presenting with symptoms for $<12$ h vs. 12-72 h. Eur Heart J. 2009;30:1322-30.

4. Weir RA, Miller AM, Murphy GE, Clements S, Steedman T, Connell JM, McInnes IB, Dargie HJ, McMurray JJ. Serum soluble ST2: a potential novel mediator in left ventricular and infarct remodeling after acute myocardial infarction. J Am Coll Cardiol 2010;55:243-50.

5. Murakami T, Kusachi S, Murakami M, Sano I, Uesugi T, Hirami R, Kajiyama A, Kondo J, Tsuji T. Time-dependent changes of serum carboxy-terminal peptide of type I procollagen and carboxy-terminal telopeptide of type I collagen concentrations in patients with acute myocardial infarction after successful reperfusion: correlation with left ventricular volume indices. Clin Chem 1998;44:2453-61.

6. Kong JB, Li QF, Yang CS, Kong DG, Wang LX. Effect of primary percutaneous coronary intervention on serum collagen biomarkers following acute myocardial infarction. Acta Cardiologica 2010;65:661-4.

7. Hørslev-Petersen K, Kim KY, Pedersen LR, Bentsen KD, Uldbjerg N, Oxlund H, Garbarsch C, Hahn EG, Schuppan D, Lorenzen I. Serum aminoterminal type III procollagen peptide: relation to biosynthesis of collagen type III in experimental induced granulation tissue in rats. Acta Pathol Microbiol Immunol Scand 1988;96:793-804.

8. Jirmar R, Pelouch V, Widimsky P, Capek J, Andel M, Prusa R, Pechova $M$. Influence of primary coronary intervention on myocardial collagen metabolism and left ventricle remodeling predicted by collagen metabolism markers. Int Heart J 2005;46:949-59.

9. Ren HZ, Zhang XS, Wang LX. Effect of coronary revascularization on serum collagen biomarkers and left ventricular remodeling in patients with acute myocardial infarction. Heart Lung 2012;41(4):344-9.

10. Sutton MG, Sharpe N. Left ventricular remodeling after myocardial infarction: pathophysiology and therapy. Circulation 2000;101:29818.

11. Poulsen SH, Host NB, Egstrup K. Long-term changes in collagen formation expressed by serum carboxyterminal propeptide of typeI procollagen and relation to left ventricular function after acute myocardial infarction. Cardiology 2001;96:45-50. 
12. Soejima H, Ogawa H, Sakamoto T, Miyamoto S, Kajiwara I, Kojima S, Hokamaki J, Sugiyama S, Yoshimura M, Suefuji H, Miyao Y, Fujimoto K, Miyagi H, Kishikawa H. Increased serum matrix metalloproteinase-1 concentration predicts advanced left ventricular remodeling in patients with acute myocardial infarction. Circ J 2003;67:301-4.
13. Manhenke C, Orn S, Squire I, Radauceanu A. Alla F, Zannad F, Dickstein $\mathrm{K}$. The prognostic value of circulating markers of collagen turnover after acute myocardial infarction. Int J Cardiol 2011;150:277-82. 